


\title{
Mehr Frauen in Führungspositionen - More women in Leadership Roles
}

\author{
Interview mit Prof. Dr. med. Monika Engelhardt, Universitätsklinikum Freiburg
} -Autorin von Dr. med. vet. Astrid Heinl, JOURNAL ONKOLOGIE 12/2020

\begin{abstract}
The high proportion of women among medical students is remarkable. Nevertheless, there are still few female doctors who reach a higher position in their professional careers. At some point in their career, it seems difficult to move up further, often due to the desire to start a family. Is it possible to overcome career obstacles for talented female scientists and reduce inequalities between women and men in medicine?
\end{abstract}

Prof. Dr. Monika Engelhardt, Freiburg, will talk about this topic.

Schon seit Jahren studieren mehr Frauen als Männer Medizin, dennoch sind Frauen immer noch seltener in Chefetagen zu finden. Woran liegt das - spielen neben Schwangerschaft und Elternzeit auch immer noch geschlechtsspezifische Vorurteile eine Rolle? Sehen Sie hier einen Wandel?

Ich sehe im Vergleich zu den vergangenen 10-20 Jahren einen Wandel: heute studieren mehr Frauen als Männer Medizin, d.h. 60\% der Studierenden sind weiblich (Abb. 1), die typischerweise auch sehr ambitioniert sind. Sie machen oft eine gute Doktorarbeit und fangen zügig an, als Assistenzärzte zu arbeiten. Es sind dann aber vor allem die Frauen, die die Karriere wegen Kindern unterbrechen und/oder ihre Arbeit reduzieren. Sie überlegen sich auch eher, ob sie weiterhin an einer Universitätsklinik verbleiben, sich habilitieren und eine leitende bzw. Chefposition anstreben sollen. Obwohl Frauen zu 60\% mit dem Medizinstudium beginnen, sind sie sehr viel seltener später in leitenden Positionen und in weniger als 10\% in Chefetagen anzutreffen, was bedauerlich ist (Abb. 1). Hier bestehen somit große geschlechtsspezifische Unterschiede. Um Frauen auf dem Karriereweg 
erfolgreich zu begleiten und zu motivieren, dauerhaft im Beruf zu verbleiben, bedarf es einer besonderen Frauenförderung. Hierfür setzen sich viele ein, wie die Familie (z.B. die Eltern), später der richtige Partner, Kollegen und im Idealfall ein(e) exzellente(r) Chef/in. Auch Frauenförderungsprogramme sind dabei hilfreich: in der Universitätsklinik Freiburg gibt es beispielsweise definierte Gender Förderungsprogramme, bei denen sich weibliche und männliche Mentoren für ihre Mentees besonders engagieren und für deren individuelle Förderung einsetzen. Letztere werden über einen definierten Zeitraum von mindestens 1-2 Jahren begleitet, was sehr gut angenommen und inzwischen von vielen Universitätskliniken angeboten wird.

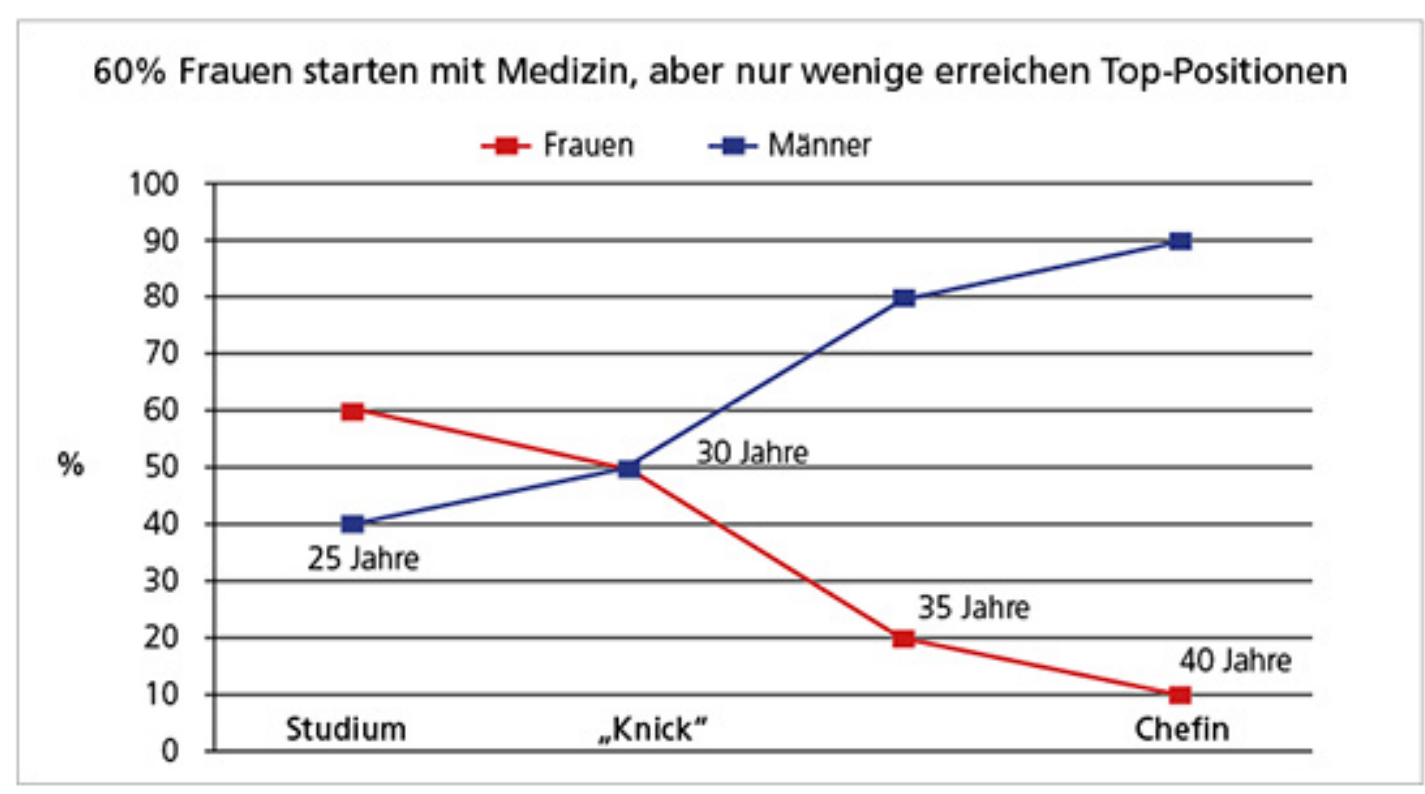

Abb. 1: Karriereverlauf von Ärztinnen mit typischem „Karriere-Knick" um das 30. Lebensjahr. (9) Mit freundlicher Genehmigung von Frou Prof. Dr. med. Gobriele Koczmorczyk, Vizeprösidentin Deurscher Ärztinnenbund 


\section{Wie viel Prozent der Lehrstühle sind derzeit von Frauen besetzt? Gibt es bevorzugte Fachgebiete wie z.B. Gynäkologie? Wie sieht es mit dem Anteil von} Frauen in der Onkologie aus?

Es gibt 36 Lehrstühle für Hämatologie und Onkologie in Deutschland, davon sind aktuell 3 $(8 \%)$ - in Kiel, Halle und Tübingen - mit einer Frau besetzt. Frauen in dieser Position sind noch deutlich unterrepräsentiert, das müssten somit mehr werden. Hämatologie und Onkologie gilt aus mehreren Gründen als „herausforderndes“ Fachgebiet: es ist emotional belastend, zudem dauert die Ausbildung lange (früher erst als Internist, anschließend erfolgte die Weiterbildung zum Hämatologen und Onkologen). Die Weiterbildung ist jetzt mit 6 Jahren bis zum Facharzt schneller möglich, daran schließen sich oft weitere Spezialisierungen, wie Palliativmedizin, an. Zudem kann man sich in dieser Zeit habilitieren, aber auch hier braucht man einen langen Atem. Anfangs sind in der Facharztzeit noch zur Hälfte Frauen und Männer, aber an der Spitze ändert sich deren Relation und man findet weniger Frauen: wir haben z.B. in Freiburg 11 Oberarztstellen, davon sind 2 mit Frauen besetzt (somit $18 \%$ statt $50 \%$ ). Man kann also feststellen: „There are many of us, but few reach the top” (Abb. 1). Warum das so ist, haben viele Publikationen zu dem Thema aufgezeigt, die verdeutlichen, dass Frauen in der Regel weniger gefördert werden, seltener exzellente Empfehlungsschreiben erhalten, in geringem Ausmaß über gute Netzwerke verfügen, selten weibliche Vorbilder haben und geringere Fördergelder bewilligt bekommen (Abb. 2). Es gibt viele Ansatzpunkte, die geändert gehören, z.B. das Bewusstsein, die o.g. Punkte anzugehen, zudem Frauenförderungsprogramme, die Frauen ermutigen, Veränderungen zu wagen, sich öfter auf höhere Positionen zu bewerben und standhaft zu bleiben, d.h. sich durchzuboxen. Die in Abbildung 3 genannten Punkte können helfen, dieses Ziel zu erreichen. 


\section{Gründe, warum Frauen seltener höhere Positionen erreichen}

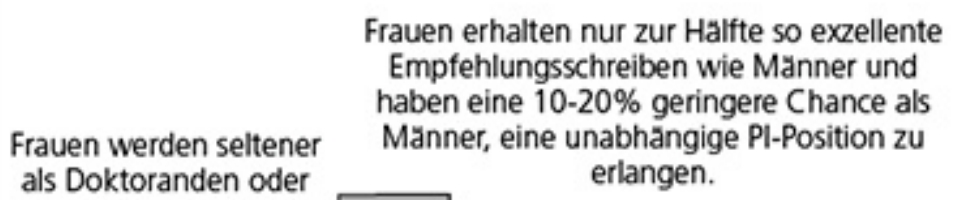
als Doktoranden oder Postdocs bei mănnlichen Pls, die $>70 \%$ der Labore innehaben, eingestellt.

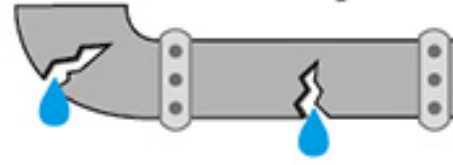

Frauen reichen generell weniger Drittmittelantrăge, Nachfolgeantrăge und gleichzeitige Antrăge ein und weisen eine geringere Wahrscheinlichkeit auf, Nachfolgeantrăge genehmigt zu bekommen als Mănner.

Frauen als Letztautoren weisen eine $6,4 \%$ geringere Chance auf, ihr Manuskript - in einem nur aus mănnlichen Gutachtern bestehenden Reviewprozess angenommen zu bekommen.

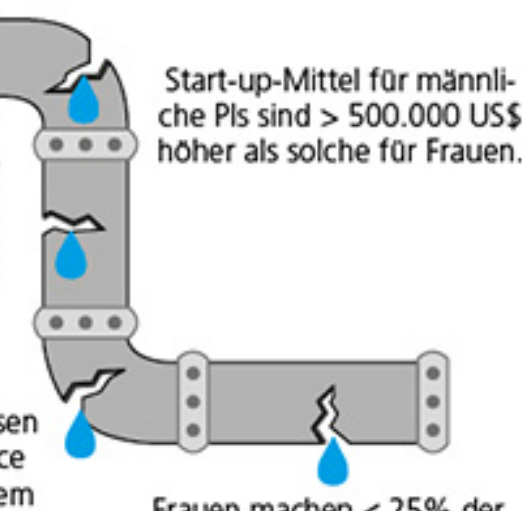

Frauen machen $<25 \%$ der Preistrăger für prominent ausgeschriebene Preise aus.

Abb. 2: Mögliche Gründe (mod. nach $(1,2)$ ). Pl-Principol Investigotor

\section{Was getan werden kann}

• Führung übernehmen

-Anerkennung der "Minderheitensteuer" (d.h. in Männerdomänen/einer von Männern geprägten Welt, eine Minderheit/Ausnahme darzustellen, die von der Umgebung nicht oder anders erwartet wird)

- Dem „Impostor-Syndrom" trotzen (psychologisches Muster, bei dem ein Individuum an eigenen Errungenschaften zweifelt und dauernde Angst hat, als „Betrüger” aufzufliegen)

- Forschung und Bewertung überdenken

- Frauenquote

-... und alle organisatorischen Aspekte, wie Abschaffung von Altersgrenzen, Verkürzung der Arbeitszeit, Organisationsoptimierung, Kinderbetreuung, ... 


\section{Familiäre Angelegenheiten haben einen großen Einfluss auf die Karrieren von} Frauen. Ist es denn aber grundsätzlich für Ärztinnen möglich, trotz Teilzeitarbeit Karriere zu machen? Würde es helfen, mehr Kinderbetreuungsangebote in den Kliniken anzubieten

Ich glaube, dass in modernen Familien Männer auch öfter bereit sind, in Teilzeit zu arbeiten, trotzdem sind es eher die Frauen, die dies über längere Zeit durchführen. Das verdeutlicht aktuell die COVID-19-Pandemie: Schulen und Kindergärten wurden geschlossen, und zuhause blieben häufig die Frauen. Bezüglich der Kinderbetreuung zeigen sich auch deutliche Unterschiede zwischen den neuen und alten Bundesländern: letztere erscheinen noch deutlich konservativer, während z.B. Jena und Dresden - nach Aussage meiner Kolleginnen - „die besten Kinderbetreuungen Deutschlands aufweisen“. Frauen müssen gut unterstützt werden, damit Arbeit und Kinderbetreuung für sie vereinbar bleibt. Schulen und Kindergärten sollten daher auch in der aktuellen Situation offenbleiben, damit Frauen und Männer ihrer Arbeit nachgehen können.

\section{Die Artikel männlicher Autoren machen den Großteil der wissenschaftlichen Publikationen aus. Woran liegt das?}

Für Publikationen und Drittmittel wird grundsätzlich viel Zeit benötigt. So wird ein Manuskript oder Drittmittelantrag sehr akribisch vorbereitet und muss bis zur Perfektion gebracht werden. Das ist ein langer und aufwändiger Prozess, den Männer einfacher zu bewältigen scheinen. Durch diesen Prozess muss aber jeder durch, da Frauen und Männer das Gleiche leisten sollten. Die Leistung der Frauen - durch deren Kinderbetreuung - wird heutzutage bei Berufungen berücksichtigt, zudem gibt es bei Berufungskommissionen Frauenbeauftragte und Mitarbeiter des wissenschaftlichen Dienstes, die diesen Punkt bei den Berufungen von Frauen genau beachten, trotzdem sind Höhe der Publikationen und Drittmittel harte Kriterien, die zwischen weiblichen und männlichen Bewerbern kompetitiv verglichen und von beiden gleichermaßen eingefordert werden.

Um Frauen auf ihrem beruflichen Weg und in ihren selbstgesteckten Zielen zu begleiten, haben Fachgesellschaften Netzwerke für die gegenseitige Frauenunterstützung gegründet: bei der Deutschen Gesellschaft für Hämatologie und Onkologie (DGHO) gibt es definierte Frauenförderungsprogramme, für die sich die DGHO engagiert. Interessierte Frauen intensivieren somit ihre Netzwerke. Bei der European Hematology Association (EHA) gibt es ähnliche Programme, ebenso bei der American Society of Hematology (ASH), American Society of Clinical Oncology (ASCO) und vielen anderen mehr. 


\section{Sind Frauen auch deshalb seltener in der Forschung zu finden, weil sie weniger Forschungsgelder bekommen und seltener befördert werden, selbst wenn Frauen dieselben Qualifikationen und Berufserfahrungen wie ihre Kollegen aufweisen?}

Auch hier ist es so, dass sich Frauen manchmal weniger zutrauen und in der Regel weniger Publikationen aufweisen. Frauen werden auch weniger Gelder zugebilligt; das alles führt dazu, dass sie weniger Förderungen erhalten (Abb. 2). Generell erfolgen Förderungen anhand objektiver Kriterien, wie z.B.: wurden die richtigen Vorarbeiten geleistet, ist ausreichend Expertise vorhanden, gibt es die richtigen Kooperationspartner und ist das geplante Forschungsfeld erfolgversprechend? Mögliche Punkte für eine Ablehnung von Forschungsgeldern sind, dass o.g. Punkte nicht oder unzureichend als erfüllt angesehen werden, zu hohe Geldsummen beantragt werden oder essenzielle Kooperationspartner fehlen. Es fließen außerdem noch andere Faktoren in die Bewilligung von Forschungsgeldern ein. So gibt es bei einer Ablehnung eines Antrags einer bekannten Forschungsgruppe mit hochkarätigen Wissenschaftlern möglicherweise massive Proteste, während dies weniger auftritt, wenn der Antrag einer Frau (die nicht protestiert), abgelehnt wird. Deshalb sind Frauen gelegentlich in einer ungünstigeren Position, u.a., weil sie nicht über ausreichend Netzwerke verfügen. Daraus entwickelt sich ein Mechanismus, dass aufgrund einer früheren Ablehnung ein erneuter Antrag wieder abgelehnt wird. Das macht es immer schwieriger für den Antragsteller. Man braucht somit ein dickes Fell, gute Ideen, viel Zeit und Energie. Man muss hartnäckig bleiben und darf sich trotz mancher Widrigkeiten nicht entmutigen lassen, da üblicherweise 70-80\% aller Anträge abgelehnt und nur 20-30\% angenommen werden. Grundsätzlich bleibt aber auffällig, dass es weniger Drittmittel für Frauen gibt (Abb. 2).

Für viele Frauen und Männer spielen auch heute die Lebensperspektive und -qualität eine große Rolle. Sie wollen ein(e) gute(r) Arzt/Ärztin sein, aber nicht jeden Tag 12-14 Stunden und noch am Wochenende arbeiten. Sie überlegen sich somit genau, ob sie noch Drittmitteleinwerbung und Publikationen bewältigen. Das hat sich im Vergleich zu geburtenstarken Jahrgängen deutlich verändert. Patientenversorgung, Lehre und Forschung (mit Publikationen, Drittmittelanträgen und Habilitation) nachzukommen, ist mit einem verkürzten Arbeitsalltag herausfordernd. Daher gibt es für Frauen und Männer jetzt z.B. „Young Scientist Programme“, bei denen auf eine egalisierte Frauenund Männerverteilung geachtet wird. Wer angenommen wird, wird von klinischen Aufgaben befreit, um sich der Wissenschaft widmen zu können und um Anträge für neue Forschungsthemen und Publikationen zu erstellen. Dadurch wird die Facharzt-Ausbildung in der Regel etwas verlängert, dieses ist aber ein wichtiges Instrument, um begabten Ärzten und Wissenschaftlern Forschung nahezubringen bzw. zu erlauben. 


\section{Ist eine Forscherkarriere mit hohem Einkommen und Prestige für Männer wichtiger als für Frauen? Oder legt die heutige Ärzte-Generation vielleicht sogar mehr Wert auf eine ausgewogene Work-Life-Balance?}

Ich würde schon sagen, dass sich das ändert, aber vielleicht ist das für Männer noch etwas wichtiger. Frauen verlassen sich heute aber nicht mehr auf den Mann als Ernährer oder achten darauf, dass der Mann ein höheres Einkommen hat, sondern können sich unabhängig von einem Partner/Ehemann versorgen. Die Work-Life-Balance spielt zudem bei beiden, Frauen und Männern, eine größere Rolle. Früher haben Ärzte z.B. sehr gern Dienste übernommen, um mehr zu verdienen. Das hat sich geändert. Ärzte sind grundsätzlich zwar überaus hilfsbereit, motiviert und engagiert, aber nicht mehr in dem Ausmaß wie früher bereit, zusätzliche Dienste zu übernehmen, sondern bevorzugen, auch einmal einen Tag frei zu haben.

\section{Untersuchungen haben gezeigt, dass Ärztinnen besser und länger zuhören und sich strenger an die Leitlinien halten. Wie unterscheiden sich Ärztinnen und Ärzte bezüglich ihrer Arbeitsweise oder der Kommunikation?}

Es gibt Publikationen, die zeigen, dass Ärztinnen sehr gut sowohl Frauen als auch Männer als Patienten betreuen, während Männer als Ärzte gegenüber weiblichen Patienten Defizite aufweisen können, weil sie z.B. weniger gut zuhören oder weniger auf die Patientinnen eingehen. Mit diesen Publikationen werden somit Gender-Unterschiede aufgezeigt. Frauen als Ärztinnen haben deshalb großes Potenzial und sind wegen ihrer Qualifikationen besonders gut für den Arztberuf geeignet. Sie sind also keinesfalls die schlechteren Mediziner. Doch Frauen leiden nicht selten unter dem sog. „Impostor-Syndrom“ (Abb. 3) und stapeln eher zu tief als zu hoch. Sie fragen sich, ob sie sich bestimmte Aufgaben überhaupt zutrauen und ob nicht vielleicht doch die Männer diese übernehmen sollten. Dieser Herausforderung sollten sich Frauen stellen und sich wie Männer präsentieren nach dem Motto „Hallo, hier bin ICH“, sonst werden sie erfahrungsgemäß unterschätzt. Das Tiefstapeln sollten Frauen somit lassen. Auch sollten Frauen nicht stets Aufgaben wie „Protokoll-Schreiben“ übernehmen, sondern dafür auch auf männliche Kollegen verweisen (Abb. 3).

Ich und viele andere werden oft gefragt, ob wir für eine Frauenquote seien. Einige Frauen und Männer mögen bedauernd darauf verweisen, dass sich dies ohne Quote mit der Zeit besser allein regeln sollte, allerdings zeigen die vergangenen Jahre, dass sich Chancengleichheit ohne Frauenquote keinesfalls automatisch regelt. Publikationen belegen zudem, dass sich Frauen erst durchsetzen und Veränderungen bewirken können, wenn sie ab einem Anteil von 30\% in Gremien vertreten sind und Verbündete finden. Seitdem bin ich für eine Frauenquote. Ohne diese Quote 
können Frauen Entscheidungen nicht relevant beeinflussen. Die Quote ist auch wichtig, um eine Frauenförderung zügiger umzusetzen. Erfreulicherweise kommt es jetzt auch zur Frauenquote für Vorstände größerer Unternehmen, was ein Durchbruch und ein wichtiger Schritt auf dem Weg der Gleichstellung und Chancengleichheit in Deutschland ist.

Auch die bereits erwähnten gegenseitigen Netzwerke sind hilfreich. Es ist sinnvoll, sich z.B. Unterstützung von einer Lehrstuhlinhaberin zu holen, denn diese Frauen sind Vorbilder, die man fragen kann, welche Maßnahmen sie ergriffen haben und welche Karriere-Tipps sie geben können, um bestimmte Ziele und Positionen zu erreichen.

Gelegentlich lässt sich beobachten, dass Frauen gegenüber Frauen strenger als gegenüber Männern agieren, deshalb gelten gemischte Teams auch als besonders erfolgversprechend. Ersteres mag daran liegen, dass Frauen oft selbst einen steinigen Karriereweg hinter sich haben und der Meinung sind, dass andere Frauen ähnliche Wege gehen sollten, um erfolgreich zu sein, was selbstverständlich individuell unterschiedlich und nicht zwingend richtig sein muss. Generell sollten Frauen aber Netzwerke pflegen, so wie dies Männer hervorragend leisten. Dazu gehört der Deutsche Ärztinnenbund, Fachvertretungen, Familie, Partner, Kollegen, Chefs und Frauenförderungsprogramme. Wenn es noch mehr dieser Personen und Programme gäbe, wäre das im Sinne vieler Frauen sehr begrüßenswert und für mehr Chancengleichheit auf dem längeren Karriereweg der Frauen ein großer Gewinn.

\section{Vielen Dank für das Gespräch!}

Dieses Interview ist meinen Eltern, Prof. Dr. Karlheinz († 22.4.2011) und Dr. Elisabeth Engelhardt, und meinem Mann, Prof. Dr. Ralph Wäsch, mit großem Dank gewidmet, die mich immer unterstützt haben, meinen Chefs, Prof. Dr. Dr. h.c. Roland Mertelsmann und Prof. Dr. Justus Duyster, die mich gefördert haben, meinen Schwestern, Brigitte Bokern und Corinna Ernst, und meinen Nichten, Teresa und Emma Bokern sowie Jana Ernst, die schon Medizin studieren oder dieses noch werden und den vielen begabten, mich fortwährend inspirierenden Kollegen, Doktoranden und Studenten, auf dass diese o.g. Netzwerke und Förderungen finden und nutzen mögen, die besonders für Frauen ermutigend und wichtig sind.

\section{Literatur:}

(1) Grogan KE. Nat Ecol Evol 2019;3(1):3-6.

(2) Gießelmann K. Dtsch Arztebl 2020;117(4): A-119/B-107/C-103. 